\title{
Impact of the ISCHEMIA Trial on Stress Nuclear Myocardial Perfusion Imaging
}

\author{
Venkatesh L. Murthy ${ }^{1}$, Timothy M. Bateman ${ }^{2}$, Wengen Chen ${ }^{3}$, Saurabh Malhotra ${ }^{4,5}$, Edward J. Miller ${ }^{6}$, Terrence D. Ruddy ${ }^{7}$ \\ and Vasken Dilsizian ${ }^{3}$ on behalf of the Society of Nuclear Medicine and Molecular Imaging Cardiovascular Council \\ ${ }^{I}$ Division of Cardiovascular Medicine and Frankel Cardiovascular Center, University of Michigan, Ann Arbor, Michigan; ${ }^{2}$ Mid \\ America Heart Institute and the Saint-Luke's Health System, University of Missouri-Kansas City, Kansas City, Missouri; ${ }^{3}$ Department \\ of Diagnostic Radiology and Nuclear Medicine, University of Maryland School of Medicine, Baltimore, Maryland; ${ }^{4}$ Division of \\ Cardiology, Cook County Health, Chicago, Illinois; ${ }^{5}$ Division of Cardiology, Rush Medical College, Chicago, Illinois; ${ }^{6}$ Section of \\ Cardiovascular Medicine, Yale School of Medicine, New Haven, Connecticut; and ${ }^{7}$ University of Ottawa Heart Institute, Ottawa, \\ Ontario, Canada
}

$\mathbf{T}$ he International Study of Comparative Health Effectiveness with Medical and Invasive Approaches (ISCHEMIA) was a randomized trial of 5,179 patients comparing the effects of optimal medical therapy versus the combination of optimal medical therapy and coronary revascularization on major adverse cardiac outcomes in patients with moderate to severe ischemia $(1,2)$. Overall, the ISCHEMIA trial found that revascularization did not lower the rates of major adverse cardiac outcomes or cardiac death. However, unmasked revascularization did improve angina symptoms and quality of life for patients with daily to monthly angina at baseline (3). There has been broad discussion and many strong proclamations of the implications of these data on the practice of clinical cardiology. Given the substantial implications on management of patients with known or suspected coronary artery disease, the Cardiovascular Council of the Society of Nuclear Medicine and Molecular Imaging, the leading professional society related to the practice of nuclear medicine imaging, would like to offer its preliminary perspective on these data.

\section{PRELIMINARY DATA AND MOTIVATION FOR THE TRIAL}

The initial impetus for this trial was observational data from a single U.S. center demonstrating that patients with ischemia affecting more than $10 \%-12.5 \%$ of the left ventricular myocardium on stress SPECT myocardial perfusion imaging who underwent early revascularization had lower mortality than those who did not $(4,5)$. A critical issue with observational data is the potential for biased referral for revascularization leading to a correlation between revascularization and outcomes without causation. The authors used propensity methods to reduce the likelihood of this type of erroneous conclusion, although only randomization can eliminate it entirely. Consequently, use of a simple heuristic that greater than $10 \%$ ischemia should drive early revascularization in suitable patients became widespread. Since the time of these studies, there have been substantial improvements in the nature and

Received May 14, 2020; revision accepted May 18, 2020.

For correspondence or reprints contact: Venkatesh L. Murthy, University of Michigan, 1338 Cardiovascular Center, 1500 E. Medical Center Dr., SPC 5873, Ann Arbor, MI 48109.

E-mail: vlmurthy@med.umich.edu

Published online Jun. 8, 2020.

COPYRIGHT @ 2020 by the Society of Nuclear Medicine and Molecular Imaging. DOI: 10.2967/jnumed.119.245399 use of optimal medical therapy as well as in revascularization devices and techniques to reduce the rate of serious complications. Further, until recently, no similar data were published from any other groups or with any other stress testing modalities. Given the costs and potential complications associated with coronary revascularization, the National Heart, Lung, and Blood Institute funded the ISCHEMIA trial to assess this practice more rigorously.

\section{STUDY DESIGN AND IMPLEMENTATION}

Performing a large and complex randomized trial such as ISCHEMIA is a massive undertaking, and the trialists and investigators deserve congratulations for completing this effort. Nonetheless, a large and complex trial, such as this, necessitates compromises in design that can have implications on interpretation. The limitations of design in the ISCHEMIA trial have been discussed in depth (6). The most serious of these is the claim that preliminary data from the earlier studies suggested that as a group, outcomes among patients with greater than 10\%-12.5\% ischemia on SPECT myocardial perfusion imaging were better with early revascularization. However, at exactly $10 \%-12.5 \%$ ischemia, outcomes were identical. Indeed, the observed survival did not actually diverge until greater than $15 \%$ ischemia was observed $(4,5)$.

To facilitate recruitment, the initial study design permitted stress testing with nuclear, echocardiographic, or MR imaging, with compromises made due to limited evaluation of some portions of the heart with nonnuclear testing (7). The initial plan was to mandate core lab confirmation of sufficient ischemia before randomization, although this requirement was lifted in the interest of improving trial flow. Furthermore, even the requirement for stress imaging was eventually lifted and patients with stress electrocardiography without imaging were permitted to be enrolled (1). There have been no preliminary data showing that this modality is able to identify patients for whom revascularization may be beneficial. Given limited sensitivity and specificity, marked differences in referral patterns, and potential for confounding by frailty, this was a major compromise. In the end, only $33.8 \%$ of the population randomized $(n=1,748)$ had imaging evidence of severe myocardial ischemia. Of these, only 970 (18.7\% of those randomized) had severe ischemia on nuclear imaging, for which there were pretrial data supporting benefit from revascularization $(4,5)$.

How serious a compromise was this? First, patients who underwent stress electrocardiography without imaging comprised a 
larger share of the trial population $(24.5 \%)$ than those for whom preliminary data that motivated this trial suggested might benefit from revascularization (namely those with moderate or greater ischemia on a stress nuclear myocardial perfusion imaging study). Further compounding this limitation, all the nonimaging patients were characterized as having "severe" ischemia, although quantification of ischemic burden on stress electrocardiography has limited data and poor calibration. This has severely limited the subgroup analysis. Indeed, the results presented showed that patients with severe ischemia had a better prognosis than those with only moderate ischemia $(12.7 \%$ vs. $13.8 \%$ estimated 4 -y event rate). Although it is tempting to conclude that this trial overturns the vast volume of data showing a strong relationship between ischemia burden and prognosis from many hundreds of thousands of patients across numerous centers, a better interpretation is that the definitions used were flawed and the trial recruitment biased.

The trial was designed as a clinical outcomes study, and not for optimization or comparison of imaging strategies. Furthermore, details of how important ancillary imaging findings such as transient cavity dilation, decrease in ejection fraction, and stress-induced symptoms were incorporated into core lab decisions have not been explained. In clinical practice, these factors are often at least as important as perfusion defect size to clinical decision making with regard to referral for angiography and revascularization. This may be reflected in the relatively high percentage of patients without symptoms who were randomized $(\sim 35 \%)$. Generally, asymptomatic patients are considered rarely appropriate for stress testing by professional-society appropriate-use criteria (8).

Another serious issue is that there was no requirement for complete revascularization or even for revascularization of territories corresponding to ischemia. There is evidence that even at quaternary medical centers, revascularization is often performed in nonischemic territories while ignoring other ischemia-causing lesions (9). Of course, with stress electrocardiography, localization of ischemia can be very challenging. It will be critical to assess the concordance between revascularization and ischemic burden to judge whether the primary hypothesis was indeed implemented in the revascularization arm.

\section{FORWARD ROLE FOR NUCLEAR STRESS TESTING}

First, the prognostic value of nuclear stress testing is extremely well established and is not challenged by this trial, which was not optimized for this question $(10,11)$. Second, nuclear methods continue to advance, with marked improvements in accuracy and reductions in radiation dosimetry. Thus far, there have been no data presented with regard to types or capabilities of cameras or to the protocols used. Unfortunately, an international survey showed that many centers are using outdated techniques and protocols with suboptimal performance. Of note, only $10 \%$ of the included perfusion studies were performed in the United States, and thus, they may not reflect optimized clinical protocols or those in use today (12).

Third, availability of cardiac nuclear imaging is broad, as is expertise in interpretation of these studies. Finally, nuclear stress testing can be performed effectively in patients with renal or lung disease, irregular cardiac rhythms, and obesity-which represent challenges to alternative testing strategies. Consequently, there will continue to be an important role for nuclear methods in the prognostic assessment of patients with known or suspected coronary artery disease and intermediate or higher clinical risk.
One limitation raised has been the rate of left main coronary stenosis in this population $(5.8 \%)$ on masked coronary CT angiography performed before randomization. This suggests a potential role for coronary CT angiography after stress testing in intermediate- to high-risk patients with moderate to large perfusion defects but also reemphasizes the importance of identifying findings on stress testing associated with high or low rates of left main coronary involvement. Several of these have already been identified, but further work would be fruitful (13-16). One important limitation is that approximately $11 \%$ of those randomized could not undergo coronary CT angiography because of reduced renal function.

Another major change will likely be a reduction in overall revascularization volumes with a primary focus on optimal medical therapy. Remaining referrals for revascularization of non-left main coronary stenosis will be increasingly focused on revascularization for symptom improvement (2). Anatomic stenosis and invasive measures of ischemia such as fractional flow reserve are unable to identify patients who accrue a symptomatic improvement with revascularization (17), although ischemia burden on stress imaging is able to do so (18-20). This is an important role for stress imaging for directing revascularization to those most likely to benefit and an advantage compared with anatomic approaches for diagnosis.

\section{CONCLUSION}

Although numerous secondary analyses will no doubt be performed to further characterize the data, this study should drive changes in clinical practice. Appropriate-use criteria should be refined to emphasize revascularization for symptomatic benefit of anginal symptoms and quality of life in most patients, although outcome improvement has not been ruled out for patients with severe degrees of ischemia. It will be important to review appropriate-use criteria for stress imaging to reemphasize focus on patients with an intermediate to high risk of coronary artery disease complications and to continue to improve the quality of implementation of nuclear stress imaging in clinical practice. Nonetheless, stress nuclear myocardial perfusion imaging will continue to have an important clinical role for the foreseeable future.

\section{DISCLOSURE}

Venkatesh Murthy, Timothy Bateman, Wengen Chen, Saurabh Malhotra, Edward Miller, and Terrence Ruddy are currently president, vice president, secretary, treasurer, vice president-elect, and immediate past president of the Cardiovascular Council of the Society of Nuclear Medicine and Molecular Imaging. Vasken Dilsizian is president of the Society of Nuclear Medicine and Molecular Imaging. Venkatesh Murthy owns stock in in General Electric and Cardinal Health and stock options in Ionetix. He has received consulting fees from Ionetix and Curium and payment for expert-witness testimony from Jubilant Draximage. He has received nonfinancial research support from INVIA Medical Imaging Solutions. He is the principal investigator on grants from the National Heart, Lung, and Blood Institute (R01HL136685) and the National Institute on Aging (R01AG05972). Timothy Bateman has received research grants from Bracco, GE Healthcare, and Jubilant Draximage; is a consultant for AIM, AstraZeneca, Curium, and GE Healthcare; receives royalties related to SPECT and PET software; and owns stock in CVIT. Terrence Ruddy has received grants from GE Healthcare and Advanced Accelerator Applications. Edward 
Miller is a consultant for GR Healthcare, Pfizer, Eidos, and Alnylam and receives research grants from Eidos, Bracco, Pfizer, and Alnylam. Saurabh Malhotra is a consultant for Akcea Therapeutics, is on the speaker's bureau for Pfizer, and receives research support from Canon Medical Systems. No other potential conflict of interest relevant to this article was reported.

\section{REFERENCES}

1. Maron DJ, Hochman JS, O'Brien SM, et al. International Study of Comparative Health Effectiveness with Medical and Invasive Approaches (ISCHEMIA) trial: rationale and design. Am Heart J. 2018;201:124-135.

2. Maron DJ, Hochman JS, Reynolds HR, et al. Initial invasive or conservative strategy for stable coronary disease. N Engl J Med. 2020;382:1395-1407.

3. Spertus JA, Jones PG, Maron DJ, et al. Health-status outcomes with invasive or conservative care in coronary disease. N Engl J Med. 2020;382:1408-1419.

4. Hachamovitch R, Hayes SW, Friedman JD, Cohen I, Berman DS. Comparison of the short-term survival benefit associated with revascularization compared with medical therapy in patients with no prior coronary artery disease undergoing stress myocardial perfusion single photon emission computed tomography. Circulation. 2003;107:2900-2907.

5. Hachamovitch R, Rozanski A, Shaw LJ, et al. Impact of ischaemia and scar on the therapeutic benefit derived from myocardial revascularization vs. medical therapy among patients undergoing stress-rest myocardial perfusion scintigraphy. Eur Heart J. 2011;32:1012-1024.

6. Murthy VL, Eagle KA. ISCHEMIA: a search for clarity and why we may not find it. Am Heart J. 2018;203:82-84.

7. Shaw LJ, Berman DS, Picard MH, et al. Comparative definitions for moderatesevere ischemia in stress nuclear, echocardiography, and magnetic resonance imaging. JACC Cardiovasc Imaging. 2014;7:593-604.

8. Wolk MJ, Bailey SR, Doherty JU, et al. ACCF/AHA/ASE/ASNC/HFSA/HRS/ SCAI/SCCT/SCMR/STS 2013 multimodality appropriate use criteria for the detection and risk assessment of stable ischemic heart disease: a report of the American College of Cardiology Foundation Appropriate Use Criteria Task Force, American Heart Association, American Society of Echocardiography, American Society of Nuclear Cardiology, Heart Failure Society of America, Heart Rhythm Society, Society for Cardiovascular Angiography and Interventions, Society of Cardiovascular Computed Tomography, Society for Cardiovascular
Magnetic Resonance, and Society of Thoracic Surgeons. J Am Coll Cardiol. 2014; 63:380-406.

9. Secemsky EA, Gallagher R, Harkness J, et al. Target vessel revascularization and territory of myocardial ischemia in patients with chronic total occlusions. J Am Coll Cardiol. 2017;70:1196-1197.

10. Shaw LJ, Iskandrian A. Prognostic value of gated myocardial perfusion SPECT. J Nucl Cardiol. 2004;11:171-185.

11. Dorbala S, Di Carli MF, Beanlands RS, et al. Prognostic value of stress myocardial perfusion positron emission tomography: results from a multicenter observational registry. J Am Coll Cardiol. 2013;61:176-184.

12. Einstein AJ, Pascual TNB, Mercuri M, et al. Current worldwide nuclear cardiology practices and radiation exposure: results from the 65 country IAEA Nuclear Cardiology Protocols Cross-Sectional Study (INCAPS). Eur Heart J. 2015; 36:1689-1696.

13. Berman DS, Kang X, Slomka PJ, et al. Underestimation of extent of ischemia by gated SPECT myocardial perfusion imaging in patients with left main coronary artery disease. J Nucl Cardiol. 2007;14:521-528.

14. Naya M, Murthy VL, Taqueti VR, et al. Preserved coronary flow reserve effectively excludes high-risk coronary artery disease on angiography. J Nucl Med. 2014;55:248-255.

15. Ziadi MC, Dekemp RA, Williams K, et al. Does quantification of myocardial flow reserve using rubidium-82 positron emission tomography facilitate detection of multivessel coronary artery disease? J Nucl Cardiol. 2012;19: 670-680.

16. Dorbala S, Hachamovitch R, Curillova Z, et al. Incremental prognostic value of gated $\mathrm{Rb}-82$ positron emission tomography myocardial perfusion imaging over clinical variables and rest LVEF. JACC Cardiovasc Imaging. 2009;2:846-854.

17. Al-Lamee R, Howard JP, Shun-Shin MJ, et al. Fractional flow reserve and instantaneous wave-free ratio as predictors of the placebo-controlled response to percutaneous coronary intervention in stable single-vessel coronary artery disease. Circulation. 2018;138:1780-1792.

18. Al-Lamee RK, Shun-Shin MJ, Howard JP, et al. Dobutamine stress echocardiography ischemia as a predictor of the placebo-controlled efficacy of percutaneous coronary intervention in stable coronary artery disease: the stress echocardiography-stratified analysis of ORBITA. Circulation. 2019;140:1971-1980.

19. Patel KK, Spertus JA, Arnold SV, et al. Ischemia on PET MPI may identify patients with improvement in angina and health status post-revascularization. J Am Coll Cardiol. 2019;74:1734-1736.

20. Dilsizian V, Erario M. Is exercise treadmill time or reduction in myocardial ischemia the appropriate primary endpoint to assess success of percutaneous coronary intervention in stable angina (ORBITA)? J Nucl Med. 2018;59:1-2. 